\title{
Ionospheric Calibration for Low Frequency Arrays using the Peeling Scheme
}

\author{
H.T. Intema ${ }^{a *}$, W.D. Cotton $^{b}$, A.S. Cohen ${ }^{c}$, I.M. van Bemmel ${ }^{a}$, H.J.A. Röttgering ${ }^{a}$ and \\ G.K. Miley ${ }^{a}$ \\ ${ }^{a}$ Leiden Observatory, Leiden University, P.O.Box 9513, NL-2300 RA, Leiden, The Netherlands \\ * Poster presenter; E-mail: intema@strw. leidenuniv.nl \\ ${ }^{b}$ National Radio Astronomy Observatory, Charlottesville, VA, USA \\ ${ }^{c}$ Naval Research Laboratory, Washington DC, USA
}

\begin{abstract}
We present the first results in our effort to calibrate low frequency $(<300 \mathrm{MHz})$ array observations that are suffering from ionospheric phase disturbances. These disturbances may vary as a function of time, frequency, antenna location and viewing direction, which causes the self-calibration technique to fail in many cases. The method used is a simplified version of the calibration scheme proposed for LOFAR. This method extrapolates the results from calibrations towards individual sources within the target field-of-view (the peeling scheme) by fitting a curved phase screen model at an arbitrary height, thus allowing for higher order phase corrections across intermediate sized $(\sim 10-50 \mathrm{~km})$ arrays like VLA-A/B and GMRT.
\end{abstract}

From planets to dark energy: the modern radio universe October 1-5 2007

University of Manchester, Manchester, UK 


\section{High Resolution and Low Frequency}

In the near future, several large radio interferometer arrays with low frequency capabilities will become operational. LOFAR, which is currently under construction in The Netherlands, will operate at frequencies below $300 \mathrm{MHz}$ with baseline lengths up to $100 \mathrm{~km}$. At these frequencies, the (local) phase corruptions due to the ionosphere are a main limiting factor in the calibration process. The field-of-view (FOV) is large (typically a few degrees or more in diameter), so there are many sources to deconvolve. Widely separated array elements look through different parts of the ionosphere. Phase errors per array element may vary across the FOV. The ionospheric phase errors can cause self-calibration to fail, because self-calibration tries to obtain only a single phase correction per array element for the whole FOV. To study the performance of one possible ionospheric calibration algorithm, we are using data from already existing arrays: the VLA at $74 \mathrm{MHz}$ with baselines up to $25 \mathrm{~km}$ in A-configuration and the GMRT with $30 \mathrm{~km}$ baselines available at $150 \mathrm{MHz}$.

\section{Peeling and the Ionosphere Model}

Ionospheric phase corruptions can vary at scales much smaller than the large FOV at low frequencies. The FOV will typically contain several bright sources. Imaging artefacts due to phase errors are most prominent around these bright sources, but may cause sidelobe confusion at large angular distance from the actual source. Possibly, there are also bright nearby outlier sources and very bright far away outliers (like Cas A, Cyg A or the Sun) that need to be taken into account. The peeling scheme is a method that suppresses these effects. It involves self-calibration on individual bright sources while suppressing the effect of all other sources, repeatedly using the following recipe (or similar):

- Subtraction of all but the brightest source from UV data, using the best model and calibration available.

- Several rounds of (phase-only) self-calibration and imaging on the brightest source.

- Subtraction of the brightest source from original UV data, using model and calibration from the previous step.

The peeling scheme produces phase corrections per array element for several viewing directions. We assume a thin layer ionosphere phase screen model at fixed height above the Earth's surface, which maps each peeling phase correction to a single pierce point in the thin layer model along the line from source to array element. Assuming smooth transitions between viewing directions, we fit a Zernike polynomial basis to the relevant domain per (fixed) time interval. The model allows for interpolation of the phase corrections to arbitrary viewing directions within the FOV. We adopted the polyhedron method for imaging, calculating one phase correction per array element per time interval for the center of each facet within the FOV. During each major clean cycle, the phase corrections for the facet are temporarily applied. 


\section{First Results}

Very recently, we have obtained the first results from the calibration technique described in the previous section by applying it to simulated and real VLA $74 \mathrm{MHz}$ data. We compare our results against the field-based calibration scheme by Cotton et al. [1], the single existing implementation of an ionospheric calibration scheme. The field-based calibration technique has been succesful in correcting most of the VLSS data sets. The field-based calibration technique differs from our method by that it uses source position offsets instead of peeling calibration results to do the model fitting, which takes place in the sky plane rather than the thin layer at fixed height that we use. The field-based calibration scheme assumes a compact array configuration $(<10 \mathrm{~km}$ baselines) so that all array elements roughly look through the same part of the ionosphere. In principle, the technique that we are testing here does not have this limitation.

Our simulated data set is based on a real $74 \mathrm{MHz}$ VLSS data set in B-configuration, consisting of a multiple point source model UV data set with 'quiet' ionospheric phase distortions applied. The phase distortions used were the actual phase corrections from the field-based calibration scheme for this particular field. Both methods perform equally well in terms of background noise level, flux retrieval, Strehl ratios and dynamic range.

Our real data sets consisted of two VLSS data sets, namely the data set that was used to generate the simulated data described above (B-configuration) with apparently quiet ionospheric conditions and a data set of a southern field (BnA-configuration) with apparently worse ionospheric conditions. In both cases, our method performs somewhat better than the field-based calibration method, improving the dynamic range with $\sim 10 \%$ in the inner half of the primary beam, while the field-based calibration outperforms our method towards the edge.

\section{Conclusions}

- The ionospheric calibration method presented here gives encouraging first results, but no drastic improvements over the existing field-based calibration by Cotton et al. [1].

- Performance of this method seems to be very dependent on the choice of model functions.

- Improvements as compared to the field-based calibration scheme may become more apparent when the new method is applied to VLA-A or GMRT data with $25-30 \mathrm{~km}$ baselines.

\section{References}

[1] W.D. Cotton, et al., 2004, Beyond the isoplanatic patch in the VLA Low-Frequency Sky Survey, SPIE 5489180 